\title{
The Dutch cohort of sildenafil users: baseline characteristics
}

\author{
P.C. SOUVEREIN, A.C.G. EgBERTS, M.C.J.M. STURKENBOOM*, E.J.H. MEULEMAN†, H.G.M. LEUfKENS \\ and J. URQUHART $\$$ \\ Department of Pharmacoepidemiology and Pharmacotherapy, Utrecht Institute for Pharmaceutical Sciences, Utrecht, *Department \\ of Epidemiology and Biostatistics, Erasmus University Rotterdam, Rotterdam, †Department of Urology, University Hospital \\ Nijmegen, Nijmegen, and $\ddagger$ Department of Epidemiology, Maastricht University, Maastricht, The Netherlands
}

Objective To identify a Dutch cohort of sildenafil users and describe their baseline characteristics.

Patients and methods Each pharmacy in The Netherlands $(n=1571)$ was asked to identify prospectively the first 20 sildenafil prescriptions in their pharmacy over 1 year, and to complete and return a registration form. The collected data included patient characteristics, the details of the sildenafil prescription (date, prescriber, number of prescriptions, dosing), and the use of co-medication by the patient in the year preceding the sildenafil prescription.

Results Data were collected from 4460 sildenafil prescriptions during the year under study, relating to 3477 individual patients. Most of the cohort had cardiovascular morbidity or diabetes. Sildenafil seems to have been used by a new, previously untreated population of patients with erectile dysfunction. In addition, 69 men were identified who could have been using nitrates and sildenafil concomitantly.

Conclusion A cohort of patients using sildenafil was identified and characterized; they appeared to be representative of sildenafil users in The Netherlands. This cohort will be followed prospectively to evaluate the medical status (particularly cardiovascular) of the patients with time.

Keywords sildenafil, cohort study, community-based, patient characteristics

\section{Introduction}

Erectile dysfunction (ED) is a common disorder among elderly men. Data from the Massachusetts Male Aging Study showed that the annual incidence of ED in men 40-69 years old was 26 per 1000 men, and increased with age and the presence of concomitant conditions like diabetes mellitus, heart disease and hypertension [1]. Current projections estimate that in 2025 about 322 million men worldwide will have $\mathrm{ED}$, an increase of nearly 170 million [2].

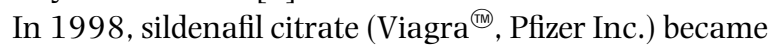
available as the first systemic (oral) drug approved for the treatment of ED. Sildenafil is an inhibitor of phosphodiesterase isotype 5 and indirectly prevents the breakdown of cGMP, the intracellular messenger that induces smooth-muscle relaxation in penile tissue and an enhanced inflow of blood. Clinical studies have shown that sildenafil is effective in ED of organic, psychological and mixed aetiologies [3]. Since its introduction to the market, sildenafil has attracted much media attention and has become a major pharmaceutical product.

Accepted for publication 1 February 2001
However, there have been concerns about the safety of sildenafil. Case reports have described patients with myocardial infarction after sildenafil administration $[4,5]$. The number of deaths spontaneously reported to the USA FDA among users of sildenafil were, when adjusted for numbers of prescriptions, substantially higher than deaths reported for locally administered prostacyclin products [6]. Pharmaco-epidemiological studies are necessary to resolve the question of whether the drug causes the observed adverse events or whether these only reflect the increased background risk from the presence of comorbidity that is associated with both the ED and the occurrence of cardiac events. One particular hazard is clearly defined, i.e. the combination of sildenafil and nitrates, which may lead to excessive reduction of blood pressure. As a result, the use of sildenafil is strictly contraindicated in patients using nitrates [7].

Despite many men using sildenafil, little is known about patterns of use in daily practice and the characteristics of these patients. Therefore, we identified and characterized a Dutch cohort of sildenafil users, with the aim of following these patients prospectively and identifying the determinants of continuation and discontinuation, and the occurrence of unwanted effects. 
In this report we present the baseline characteristics of the cohort.

\section{Patients and methods}

Sildenafil was introduced to the Dutch market in September 1998; in January 1999, we invited all 1571 Dutch pharmacies to participate in the formation of a national cohort of sildenafil users. As most patients in The Netherlands designate a single pharmacy to fill prescriptions from GPs or medical specialists, dispensing histories provide an essentially complete account of each patient's exposure to prescription drugs over time [8]. Each pharmacy was asked to identify prospectively the first 20 sildenafil prescriptions received by the pharmacy within a maximum period of 1 year.

The collected data from the cohort members included patient characteristics, the details of the sildenafil prescription and the use of co-medication by the patient in the year preceding the prescription. Specific data items were gender, date of birth and residential postal code of the patient, whether or not the patient was a regular client in the pharmacy, whether the prescription for sildenafil was the first one registered for that patient, the type of prescriber, the dose and number of tablets prescribed, whether the drug was eventually dispensed to the patient, and whether costs were covered by the patient's health insurer. The items about co-medication focused on the use of drugs for the treatment of ED, cardiovascular disease, and drugs indicated for the treatment of $\mathrm{BPH}$, diabetes mellitus, depression and gastrointestinal disorders. Concurrent use (indicating that the theoretical end-date of drug use overlapped with the start date of sildenafil prescription) was distinguished from non-concurrent use (indicating that the drug was dispensed during the year before the sildenafil prescription, but that the theoretical end-date did not overlap with the prescription date for sildenafil).

\section{Data analysis}

Patient characteristics were described and compared between age groups $(<40, \geqslant 40$ years $)$ and between patients with or with no previous ED treatment. Students' $t$-test was used to compare continuous variables between groups and the chi-square test to compare categorical variables. Prevalence rate ratios (RR) and 95\% CI were calculated to quantitatively evaluate subgroup differences. Analyses involving the history of prescription drug use were restricted to regular patients in the pharmacy, as the quality of the data for incidental pharmacy visitors cannot be guaranteed.

\section{Results}

Data were retrieved for 4460 sildenafil prescriptions during the 1-year study period; complete information about patient characteristics and the exact date of the sildenafil prescription was available in 4194 records (94\%), giving a cohort of 3477 individual patients. Table 1 shows the demographic and sildenafil-related characteristics for the patients (all men but one) and predominantly aged $40-70$ years $(80 \%)$. The regional origin of the study subjects is shown in Fig. 1; most of the patients lived in the more urban western part of The Netherlands (1512, $43.9 \%)$, whereas only $4.9 \%$ of them lived in the rural north.

For 2101 patients $(61.2 \%)$ cohort entry was related to the first prescription for sildenafil. For most the prescriber was a GP $(2778,80.0 \%)$ or urologist $(479,13.8 \%)$. Most first-time users received the recommended starting dose of $50 \mathrm{mg}$. Of the cohort members, $358(10 \%)$ were incidental rather than regular visitors to the pharmacy where the sildenafil prescription was filled. Two-thirds of the incidental visitors $(237,66.2 \%)$ visited a pharmacy that was in the municipality of their residence, while the others went to pharmacies located in another city. There

Table 1 Characteristics of patients within the Dutch cohort of sildenafil users $(n=3477)$

\begin{tabular}{lc}
\hline Characteristic & Number $(\%)^{*}$ \\
\hline Male & $3476(99.97)$ \\
Female & $1(0.03)$ \\
Mean (sD) age, years & $57(12)$ \\
Age group, $n$ (\%) & \\
$\quad<40$ & $258(7.4)$ \\
$40-49$ & $538(15.5)$ \\
$50-59$ & $1185(34.1)$ \\
$60-69$ & $988(28.4)$ \\
$70-79$ & $448(12.9)$ \\
$\geq 80$ & $60(1.7)$ \\
First prescription & \\
Yes & $2101(61.2)$ \\
Dose, mg & \\
25 & $681(19.6)$ \\
50 & $2282(65.7)$ \\
100 & $508(14.6)$ \\
Type of prescriber & \\
GP & $2778(80.0)$ \\
Urologist & $479(13.8)$ \\
Psychiatrist & $21(0.6)$ \\
Gynaecologist & $11(0.3)$ \\
Other & $183(5.3)$ \\
Number of tablets (median, 95\% CI) & $4(4-12)$ \\
Costs covered by HIC $\dagger$ & \\
Yes & $9(0.3)$ \\
\hline$*$ Because some observations are missing the total is not always $3477 ;$ \\
$\dagger$ HIC, health insurance company. & \\
& \\
\hline & \\
& \\
&
\end{tabular}


was no difference in age between irregular and regular clients.

Information about the use of prescription drugs in the year before the sildenafil prescription is summarized in Table 2. There were few patients with a history of

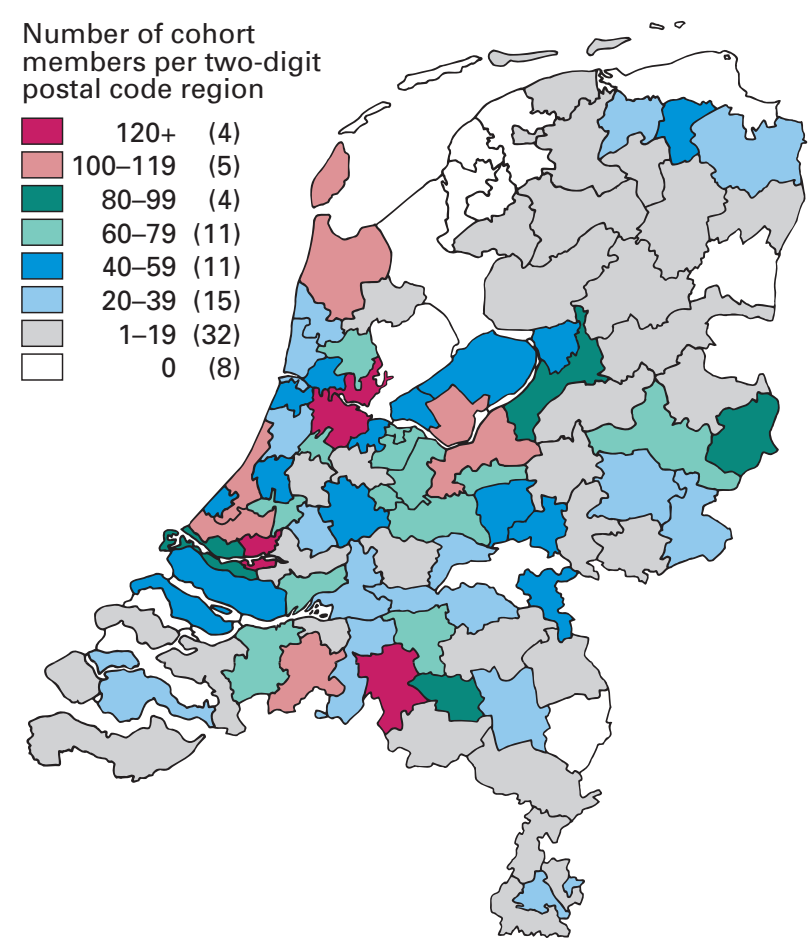

Fig. 1. The geographical distribution of patients in the Dutch cohort of sildenafil users. treatment for ED; only 195 men (6.3\%) recorded ever using yohimbine, alprostadil, or the combination of phentolamine/papaverine, or pharmacy-made preparations for cavernosal injection. The use of cardiovascular medication was widespread; at the time of cohort entry, 997 patients (32.4\%) were either current users of such drugs or had used them in the year before the prescription of sildenafil. $\beta$-blockers $(10.5 \%)$ and calcium-channel blockers $(9.8 \%)$ were the most frequently used drugs among the cohort members, while $24.0 \%$ (738) had a history of using other unspecified cardiovascular medications. The ratio between concurrent and non-concurrent users of the products listed suggests the presence of chronic cardiovascular morbidity and longterm use of these medications. Nitrates were used by 139 $(4.5 \%)$ cohort members; surprisingly, for 69 patients $(2.2 \%)$ sildenafil was prescribed while the patient was concurrently treated with nitrates.

In all, 418 (13.6\%) cohort members had a history of anti-diabetic drug use. On the date of cohort entry, 145 patients $(4.7 \%)$ were using antidepressants concomitantly and 89 patients (2.9\%) had used antidepressants in the year before. The prevalence of gastrointestinal drug use was high, with 472 men (15.4\%) being either current or former users on the sildenafil prescription date. Drugs indicated for the treatment of $\mathrm{BPH}$ were used by 183 men $(6.0 \%)$, of whom $115(3.8 \%)$ were current users. Oral anticoagulants were, or had been used on the prescription date, by $118(3.8 \%)$ and $23(0.7 \%)$ of the cohort members, respectively.

Table 2 The history of drug use on the sildenafil prescription date or during the year before (ever use), on the sildenafil prescription date only (concurrent use), and in the year before the sildenafil prescription date only (non-concurrent use) for regular pharmacy visitors only $(n=3100)$

\begin{tabular}{lrrr}
\hline Medication & Ever use & Concurrent use & Non-concurrent use \\
\hline Drugs used in ED treatment & $195(6.3)$ & $35(1.1)$ & $162(5.3)$ \\
$\quad$ Yohimbine & $23(0.7)$ & $5(0.2)$ & $18(0.6)$ \\
$\quad$ Phentolamine/papaverine & $166(5.4)$ & $26(0.8)$ & $140(4.5)$ \\
$\quad$ or alprostadil & $20(0.7)$ & $8(0.3)$ & $12(0.3)$ \\
$\quad$ Pharmacy-made for injection & $997(32.4)$ & $873(28.4)$ & $245(8.0)$ \\
Cardiovascular drugs & $139(4.5)$ & $69(2.2)$ & $70(2.3)$ \\
$\quad$ Nitrates & $325(10.5)$ & $250(8.1)$ & $75(2.4)$ \\
$\quad 3$-blocker & $302(9.8)$ & $256(8.3)$ & $46(1.5)$ \\
$\quad$ Calcium-channel blocker & $738(24.0)$ & $645(21.0)$ & $93(3.0)$ \\
$\quad$ Other & $472(15.4)$ & $299(9.7)$ & $173(5.6)$ \\
Other drugs & $176(5.7)$ & $166(5.4)$ & $10(0.3)$ \\
$\quad$ Gastrointestinal & $290(9.4)$ & $264(8.5)$ & $26(0.8)$ \\
Insulin & $141(4.6)$ & $118(3.8)$ & $23(0.7)$ \\
Oral anti-diabetics & $234(7.6)$ & $145(4.7)$ & $89(2.9)$ \\
Oral anticoagulants & $183(6.0)$ & $115(3.8)$ & $68(2.2)$ \\
Antidepressants & & \\
BPH & & & \\
\hline
\end{tabular}


Patients $\geqslant 40$ vs $<40$ years of age

Only $7.4 \%$ of the patients were $<40$ years old; sildenafil was prescribed more often by a psychiatrist, gynaecologist or other physician in the younger patients, rather than a urologist, and the daily dose diverged more from $50 \mathrm{mg}$ in the younger users than in those $\geqslant 40$ years old $(P<0.05)$. Concomitant drug use in younger patients was uncommon, with only $3.7 \%$ of these patients having a history of any cardiovascular drug use, compared with $34.6 \%$ of the patients aged $\geqslant 40$ years (Table 3 ). The same applied to the use of other drugs, with the exception of antidepressants, which were used significantly more by patients under 40 years $(14.1 \%)$ than by those aged $\geqslant 40$ years (Table 3 ).

\section{History vs no history of use of other ED drugs}

Patients with a history of using other drugs for ED (195) before cohort entry and patients with no such history (2877) were compared. GPs had issued $82 \%$ of all sildenafil prescriptions in 'new' ED patients, but only $60 \%$ of the prescriptions in men who had been treated with other drugs for ED before. 'Known' patients with ED were more likely to obtain their sildenafil prescription from a urologist (32\% vs 12\% in 'new' patients); such patients were also prescribed higher doses than patients having no history of ED treatment $(P<0.001)$.

The use of cardiovascular and other drugs among men with or with no treatment background for ED before the sildenafil prescription is shown in Table 3. 'Known' patients with ED used significantly more cardiovascular drugs than patients who started sildenafil with no history of other ED treatment ( $42.8 \%$ vs $31.7 \%$, respectively). For individual products (nitrates, $\beta$-blocking agents and calcium-channel blockers) the RR was higher but not statistically significantly so.

The use of other drugs (antidepressants excluded) was also more prevalent in the group of sildenafil users with a history of treatment for ED. Gastrointestinal medication was used by $39(20.1 \%)$ of the 'known' patients and by $431(15.1 \%)$ of the 'new' patients (Table 3). Patients with a history of ED treatment were twice as likely to use insulin than patients with no earlier treatment.

\section{Discussion}

Most of this Dutch cohort of 3477 patients using sildenafil citrate had cardiovascular morbidity or diabetes. In addition, 69 patients were identified who could have used nitrates and sildenafil concomitantly. Few patients using sildenafil had a history of using other drugs to treat ED. Therefore, sildenafil seems to have been used by a new, previously untreated population of patients with ED.

Although there has been much publicity surrounding sildenafil, data on the use of sildenafil in routine daily clinical practice, and the characteristics of sildenafil users in a community setting, are largely unknown. The present cohort was identified with the aim of following these patients with time, identifying possible unwanted events and evaluating hypotheses on the effects of sildenafil in normal use.

About $80 \%$ of the present cohort were $40-70$ years old; this is in line with data from the Massachusetts Male Aging Study which showed that the annual

Table 3 Comparison of drug use between patients aged $\geq 40$ years $(n=2879)$ and $<40(n=221)$, and between patients with $(n=195)$ or with no $(n=2877)$ history of non-concurrent use of other drugs indicated for the treatment of ED (phentolamine/papaverine, alprostadil, yohimbine, pharmacy-made preparations for cavernosal injection), for regular pharmacy visitors only

\begin{tabular}{|c|c|c|c|c|c|c|}
\hline Type of drug & $\begin{array}{l}\geq 40 \text { years } \\
\text { No. }(\%)\end{array}$ & $\begin{array}{l}<40 \text { years } \\
\text { No. }(\%)\end{array}$ & $\begin{array}{l}\text { Prevalence RR } \\
(95 \% \mathrm{CI})\end{array}$ & $\begin{array}{l}\text { History } \\
\text { No. (\%) }\end{array}$ & $\begin{array}{l}\text { No history } \\
\text { No. }(\%)\end{array}$ & $\begin{array}{l}\text { Prevalence RR } \\
(95 \% \mathrm{CI})\end{array}$ \\
\hline Cardiovascular drugs & 989 (34.6) & $8(3.7)$ & $9.5(4.8-18.7)$ & $83(42.8)$ & 909 (31.7) & $1.4(1.1-1.6)$ \\
\hline Nitrates & $139(4.8)$ & - & - & $14(7.3)$ & $125(4.3)$ & $1.7(1.0-2.8)$ \\
\hline$\beta$-blocker & $322(11.2)$ & $3(1.4)$ & $8.2(2.7-25.3)$ & $23(11.9)$ & $302(10.5)$ & $1.1(0.8-1.8)$ \\
\hline Calcium-channel blocker & $300(10.5)$ & $2(0.9)$ & $11.5(2.9-45.9)$ & $26(13.4)$ & $274(9.5)$ & $1.4(1.0-2.1)$ \\
\hline Other & $733(25.6)$ & $5(2.3)$ & $11.3(4.7-26.9)$ & $61(31.4)$ & $673(23.5)$ & $1.3(1.1-1.7)$ \\
\hline \multicolumn{7}{|l|}{ Other drugs } \\
\hline For ED & $187(6.6)$ & $8(3.7)$ & $1.8(0.9-3.6)$ & - & - & - \\
\hline Gastrointestinal & $454(15.9)$ & $18(8.2)$ & $1.9(1.2-3.1)$ & $39(20.1)$ & $431(15.1)$ & $1.3(1.0-1.8)$ \\
\hline Insulin & $172(6.0)$ & $4(1.8)$ & $3.3(1.2-8.8)$ & $22(11.4)$ & $153(5.3)$ & $2.1(1.4-3.3)$ \\
\hline Oral anti-diabetics & $287(10.0)$ & $3(1.4)$ & $7.4(2.2-22.8$ & $21(10.9)$ & $265(9.2)$ & $1.2(0.8-1.8)$ \\
\hline Oral anticoagulants & $140(4.9)$ & $1(0.5)$ & $10.8(1.5-76.8)$ & $12(6.2)$ & $129(4.5)$ & $1.4(0.8-2.5)$ \\
\hline Antidepressants & $203(7.1)$ & $31(14.1)$ & $0.5(0.4-0.7)$ & $12(6.2)$ & $222(7.7)$ & $0.8(0.5-1.4)$ \\
\hline $\mathrm{BPH}$ & $180(6.3)$ & $3(1.4)$ & $4.6(1.5-14.4)$ & $16(8.4)$ & $167(5.8)$ & $1.4(0.9-2.3)$ \\
\hline
\end{tabular}


incidence of ED per 1000 men increased from 12 new cases for men aged 40-49 to 46 new cases for men 60-69 years old [1]. In men aged $>70$ years the annual incidence is likely to increase further. The number of men in this age range is limited in the present cohort; Mulcahy [9] noted that few men aged $>70$ years seek treatment for erectile problems, possibly because only half of men aged $>70$ years are likely to be sexually active [10]. Epidemiological data have shown that cardiac disease, hypertension and diabetes are associated with the development of $\mathrm{ED}$ $[1,11]$. Vascular disease is thought to be the most common cause of organic ED [12]. Besides atherosclerotic occlusion of penile vessels, there is evidence that vascular disease is associated with structural damage to smooth muscle in the corpus cavernosum [12]. Greenstein et al. [13] found a correlation between the severity of ischaemic coronary disease and ED.

In the present study prescription drug use was used as a marker of comorbidity; $32 \%$ of sildenafil users were also using cardiovascular drugs. Furthermore, antidiabetic medication (insulin and/or oral anti-diabetics) was used by $13.6 \%$ of the cohort. These findings are comparable with the results from a prospective cohort study by Marks et al. [14], who assessed the efficacy of sildenafil in the first 100 men with a sildenafil prescription in a urology practice setting. They found that $35 \%$ of their population (mean age 64 years, sD 11) had hypertension, $30 \%$ coronary artery disease and $10 \%$ diabetes at baseline.

The administration of sildenafil is contraindicated in patients using organic nitrates, as sildenafil potentiates the hypotensive effect of these drugs $[7,15]$. In the present study, 69 patients $(2.2 \%)$ used nitrates on the day that sildenafil was prescribed. In five instances it was possible to trace signs of communication between the pharmacist and the prescriber, or approval of the prescriber about the combination of both drugs. Supplying such additional information on the registration form was optional and consequently, the incidence might be underestimated.

The use of antidepressants was twice as frequent among patients aged $<40$ years than among those aged $>40$. This result is in accordance with the study of Slag et al. [16], who found that psychogenic $\mathrm{ED}$ was more prevalent in younger men $(70 \%$ of patients with ED aged < 35 years $)$ than in older men $(10 \%$ of patients with ED aged > 50 years). Also, Karadeniz et al. [17] found, in a study designed to evaluate the aetiology of $\mathrm{ED}$ in patients aged $<40$ years, that $58 \%$ of patients aged $\leqslant 35$ had psychogenic ED. However, Donatucci and Lue [18], in a retrospective review of 100 men <40 years old, found that only $13 \%$ had psychogenic ED. The higher frequency of antidepressant use among patients aged $<40$ years could therefore also be interpreted as being the result of the disappointment, dissatisfaction, and unhappiness associated with ED in a subgroup of patients where ED (irrespective of its aetiology) is relatively unexpected.

Few patients had used alternative drugs for treating ED before starting sildenafil. This suggests that the introduction of an oral agent for ED has considerably reduced the barrier to seek medical advice for problems with erectile function.

In the present cohort, 183 men $(6.0 \%)$ were currently using drugs for the treatment of $\mathrm{BPH}$, or had used such drugs in the year before the sildenafil prescription date. The co-occurrence of BPH and sexual dysfunction is to be expected, as the prevalence of both conditions increases with age. Frankel et al. [19] found that reduced rigidity of the penis and LUTS were associated in both a community population and in patients attending a urological clinic. Uygur et al. [20] investigated the effect of several BPH treatments on ED in a 6-month prospective study and found that finasteride was associated with an impairment in erectile function, whereas the $\alpha$-blocker doxazosin was not.

All data in the present study were retrieved by the pharmacist from their computerized data files. We attempted to standardize the compilation of data by means of clear instructions and data entry protocols. Although the study was population-based, not all pharmacies responded. To verify whether the patients in the cohort are a valid sample of sildenafil users in The Netherlands, the results were compared with data from the Foundation for Pharmaceutical Statistics (SFK), who hold a pharmacy-based registry containing 1100 pharmacies $(70 \%)$ in the Netherlands. Both the geographical distribution and level of urbanization of pharmacies with sildenafil prescriptions in the SFK-database was similar to the present data. Furthermore there was no difference in age, cardiovascular drug use, or use of other prescription drugs between sildenafil users in the SFK-database and in the present cohort. Given that this information is comparable we conclude that the patients in the present cohort are representative of sildenafil users in The Netherlands.

The cohort will be followed up prospectively, obtaining detailed information on prescription drug use from the pharmacies' computerized medication databases and specific medical information from medical records. For every patient at least 1 year of follow-up is available from the date of cohort entry. The patterns of use of sildenafil with time will be assessed, to determine the frequency and determinants of (dis)continuation and the frequency of beneficial or adverse outcomes (e.g. cardiovascular). 


\section{Acknowledgements}

The authors thank all participating pharmacists for their invaluable cooperation with the study. We also thank the Foundation of Pharmaceutical Statistics for kindly providing data for validation purposes.

\section{References}

1 Johannes CB, Araujo AB, Feldman HA, Derby CA, Kleinman $\mathrm{KP}$, McKinlay JB. Incidence of erectile dysfunction in men 40-69 years old: longitudinal results from the Massachusetts Male Aging study. J Urol 2000; 163: 460-3

2 Ayta IA, McKinlay JB, Krane RJ. The likely worldwide increase in erectile dysfunction between 1995 and 2025 and some possible policy consequences. BJU Int 1999; 84: 50-6

3 Goldstein I, Lue TF, Padma-Nathan H, Rosen RC, Steers WD, Wicker PA. Oral sildenafil in the treatment of erectile dysfunction. Sildenafil Study Group. N Engl J Med 1998; 338: 1397-404

4 Arora RR, Timoney M, Melilli L. Acute myocardial infarction after the use of sildenafil. N Engl J Med 1999; 341: 700

5 Feenstra J, van Drie-Pierik RJ, Lacle CF, Stricker BH. Acute myocardial infarction associated with sildenafil. Lancet 1998; 352: 957-8

6 Mitka M. Some men who take Viagra die - why? JAMA 2000; 283: 590-3

7 Webb DJ, Freestone S, Allen MJ, Muirhead GJ. Sildenafil citrate and blood-pressure-lowering drugs: results of drug interaction studies with an organic nitrate and a calcium antagonist. Am J Cardiol 1999; 83: 21C-28C

8 Leufkens HGM, Urquhart J. Automated record linkage and other approaches to pharmacoepidemiology in The Netherlands. In Strom BL ed. Pharmacoepidemiology, 2nd edn. Chichester: John Wiley \& Sons, 1994: 231-44

9 Mulcahy JJ. Erectile dysfunction - is the incidence increasing? J Urol 2000; 163: 471

10 Bosch R, Groeneveld F, Bohnen A, Prins A, Hop W. Erectile dysfunction in a community-based sample of men aged 50-75 years: prevalence and somatic and social risk factors. Eur J Urol 1999; 35: A262

11 Feldman HA, Goldstein I, Hatzichristou DG, Krane RJ, McKinlay JB. Impotence and its medical and psychosocial correlates: results of the Massachusetts Male Aging Study. J Urol 1994; 151: 54-61

12 Jackson G. Erectile dysfunction and cardiovascular disease. Int J Clin Pract 1999; 53: 363-8

13 Greenstein A, Chen J, Miller H, Matzkin H, Villa Y, Braf Z. Does severity of ischemic coronary disease correlate with erectile function? Int J Impot Res 1997; 9: 123-6

14 Marks LS, Duda C, Dorey FJ, Macairan ML, Santos PB. Treatment of erectile dysfunction with sildenafil. Urology 1999; 53: 19-24

15 Cheitlin MD, Hutter AM Jr, Brindis RG et al. Use of sildenafil (Viagra) in patients with cardiovascular disease. Technology and Practice Executive Committee. Circulation 1999; 99: 168-77

16 Slag MF, Morley JE, Elson MK et al. Impotence in medical clinic outpatients. JAMA 1983; 249: 1736-40

17 Karadeniz T, Topsakal M, Aydogmus A, Basak D. Erectile dysfunction under age 40: etiology and role of contributing factors. Digital Urol J 1998 (http://www.duj.com)

18 Donatucci CF, Lue TF. Erectile dysfunction in men under 40: etiology and treatment choice. Int J Impot Res 1993; 5: 97-103

19 Frankel SJ, Donovan JL, Peters TI et al. Sexual dysfunction in men with lower urinary tract symptoms. J Clin Epidemiol 1998; 51: 677-85

20 Uygur MC, Gur E, Arik AI, Altug U, Erol D. Erectile dysfunction following treatments of benign prostatic hyperplasia: a prospective study. Andrologia 1998; 30: 5-10

\section{Authors}

P.C. Souverein, MSc, Epidemiologist.

A.C.G. Egberts, PhD, Professor of Clinical Pharmacoepidemiology.

M.C.J.M. Sturkenboom, PhD, Associate Professor of Pharmacoepidemiology.

E.J.H. Meuleman, MD, Urologist.

H.G.M. Leufkens, PhD, Professor of Pharmacoepidemiology.

J. Urquhart, MD, Professor of Epidemiology.

Correspondence: P. Souverein, Department of Pharmacoepidemiology \& Pharmacotherapy, Utrecht Institute for Pharmaceutical Sciences, PO Box 8002, 3508 TB Utrecht, The Netherlands.

e-mail: p.c.souverein@pharm.uu.nl 